\title{
Re: Esposito et al.: To mask or not to mask children to overcome COVID-19
}

\author{
Kaifeng $\operatorname{Jin}^{1}\left(\mathbb{D} \cdot \operatorname{Jinjin} \mathrm{Min}^{2} \cdot\right.$ Xiuming Jin ${ }^{2}$ \\ Received: 22 May 2020 / Revised: 22 May 2020 / Accepted: 13 June 2020 / Published online: 20 June 2020 \\ (C) Springer-Verlag GmbH Germany, part of Springer Nature 2020
}

To the Editor,

We are very happy to see that Esposito et al. raised an important question about children wearing masks[1]. They obtained a meaningful conclusion that the universal use of facial masks seemed necessary when people went out.

We applaud the authors for a major endeavor. We totally agree that children younger than 2 years of age do not wear any type of mask because of the small airways and risk of suffocation. However, several issues are worth supplemental noting for children over 3 years old. First, children's education. Before wearing masks, parents must have good communication with children. Children should be told how to wear and take off the mask correctly and do not touch the mask while wearing. Second, where to wear masks. In some public places that full of crowds with poor ventilation, children are recommending to wear masks. But in areas with low density of people, it may not be necessary to wear masks. Third, some interventions to improve feasibility, safety, and efficacy of wearing masks for children. Children need to take off their masks to breathe in fresh air after wearing masks for a certain amount of time, like $1 \mathrm{~h}$. Moreover, they should avoid wearing masks to do exercise, especially with N95. According to the news reported by China at least three children died of asphyx- ia after PE due to wearing N95 masks during convid-19 pandemic. Fourth, masks selection and modification for children. Surgical masks may be the first choice for children's daily protection. Only high-risk children are encouraged to wear an N95 mask. The space between the surgical masks and children's mouth and nose is smaller than adults due to the lower nose bridge (Fig. 1f). The surgical mask should be modified to make it more suitable for children. Here, we suggest a modified method for surgical mask (Fig. 1). This modified mask can not only fit snugly on their face, but also decreases the risk of contaminated air access.

The number of children with COVID-19 has also increased significantly [2]. Children of all ages appeared susceptible to COVID-19, and there was no significant sex difference [3]. Due to children are not just a reduced version of adults, preventing children from COVID-19 transmission is a difficult and important task. Only combining all preventive measures, children can get maximum protection from infection. The behaviors of mask-wearing and hand-washing among children were influenced by age, gender, and other factors; therefore, parents should make efforts of behavior guidance whereas governments should enlarge medium publicity [4].

Communicated by Peter de Winter

Kaifeng Jin

kjin01@qub.ac.uk

1 China Medical University-The Queen's University of Belfast Joint College, 77 Puhe Road, Shenyang 110136, China

2 Eye Center, Affiliated Second Hospital, School of Medicine, Zhejiang University, 88 Jiefang Road, Hangzhou 310009, China 

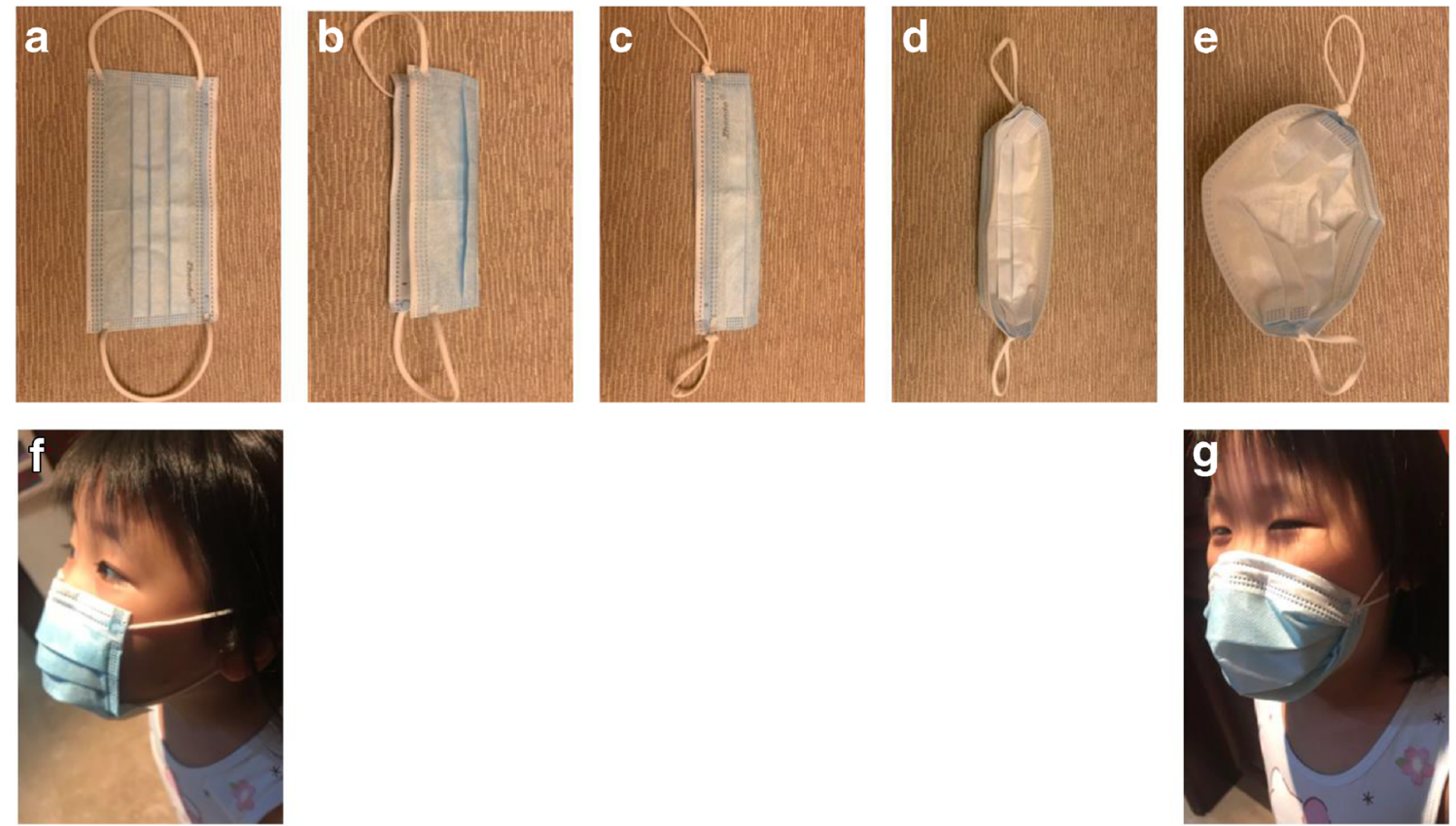

Fig. 1 The procedure of modifying surgical mask. modified surgical mask. a Normal surgical mask. b Fold the mask in half. $\mathbf{c}$ Tie the ropes up at the root of a mask. d Tuck the edges of the mask into the inner

surface. f Open modified mask. f Wear normal surgical mask. g Wear modified surgical mask

\section{Compliance with ethical standards}

Conflict of interest We declare that we have no financial and personal relationships with other people or organizations that can inappropriately influence our work. There is no professional or other personal interest of any nature or kind in any product, service, and/or company that could be construed as influencing the position presented in, or the review of, the manuscript entitled.

Competing financial interests No competing financial interests exist for any author.

\section{References}

1. Esposito S, Principi N (2020) To mask or not to mask children to overcome COVID-19. Eur J Pediatr:1-4 [published online ahead of print, 2020 May 9]. https://doi.org/10.1007/s00431-020-03674-9

2. She J, Liu L, Liu W (2020) COVID-19 epidemic: disease characteristics in children. J Med Virol 92:747-754. https://doi.org/10.1002/ jmv. 25807

3. Dong Y, Mo X, Hu Y, Qi X, Jiang F, Jiang Z, Tong S (2020) Epidemiology of COVID-19 Among Children in China. Pediatrics: e20200702. https://doi.org/10.1542/peds.2020-0702

4. Chen X, Ran L, Liu Q, Hu Q, Du X, Tan X (2020) Hand Hygiene, Mask-Wearing Behaviors and Its Associated Factors during the COVID-19 Epidemic: A Cross-Sectional Study among Primary School Students in Wuhan, China. Int J Environ Res Public Health 17(8):E2893. Published 2020 Apr 22. https://doi.org/10.3390/ ijerph17082893

Publisher's note Springer Nature remains neutral with regard to jurisdictional claims in published maps and institutional affiliations. 